\title{
Simulation of Flow Through Breach in Leading Edge at Mach 24
}

\author{
Peter A. Gnoffo* and Stephen J. Alter \\ NASA Langley Research Center, Hampton, VA 23681-0001
}

\begin{abstract}
A baseline solution for CFD Point 1 (Mach 24) in the STS-107 accident investigation was modified to include effects of holes through the leading edge into a vented cavity. The simulations were generated relatively quickly and early in the investigation by making simplifications to the leading edge cavity geometry. These simplifications in the breach simulations enabled: (1) a very quick grid generation procedure; and (2) high fidelity corroboration of jet physics with internal surface impingements ensuing from a breach through the leading edge, fully coupled to the external shock layer flow at flight conditions. These simulations provided early evidence that the flow through a two-inch diameter (or larger) breach enters the cavity with significant retention of external flow directionality. A normal jet directed into the cavity was not an appropriate model for these conditions at CFD Point 1 (Mach 24). The breach diameters were of the same order or larger than the local, external boundary-layer thickness. High impingement heating and pressures on the downstream lip of the breach were computed. It is likely that hole shape would evolve as a slot cut in the direction of the external streamlines. In the case of the six-inch diameter breach the boundary layer is fully ingested.
\end{abstract}

\section{Introduction}

$\mathrm{T}$

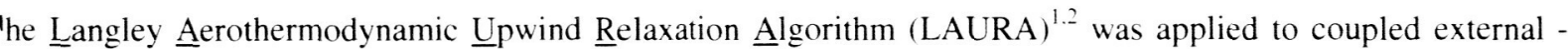
internal flow simulations for the Columbia investigation. For the case of leading edge damage, the simulations were generated to provide relatively high fidelity analyses of mass and energy ingestion rates through a breach as a function of hole size. They provided details of jet orientation and structure emanating from the breach into a vented cavity. These results were used to initialize engineering analyses of mass and energy flow through the internal structure of the wing and to better define the severity of the environment associated with the impinging jet.

Previous simulations of STS 1,2 and 5 with LAURA to resolve questions of a pitching moment anomaly ${ }^{3}$ (STS 1) and to validate heating predictions ${ }^{4.5}$ were published in the AIAA Journal of Spacecraft and Rockets in 1994. Some of this material was also presented in an ITAR restricted document with more detail and dimensions. ${ }^{6}$ There is no experimental or flight validation of the flow through the breach. The simulations were generated relatively quickly and early in the investigation on the NAS Cray SV1 because simplifications were made to the leading edge cavity geometry. These simplifications enabled (1) a very quick grid generation procedure; and (2) high fidelity corroboration of jet physics with internal surface impingements ensuing from a breach through the leading edge, fully coupled to the external shock layer flow at flight conditions.

\section{Software}

LAURA is a high fidelity analysis tool, specialized for hypersonic re-entry physics, utilizing state-of-art algorithms for computational fluid dynamic (CFD) simulations. Key elements of LAURA include Roe's averaging ${ }^{7}$ and Yee's Total Variation Diminishing (TVD) ${ }^{8}$ formulation of second-order, inviscid flux. Yee's TVD formulation has been found to be exceptionally robust and Courant-number-independent using point-implicit relaxation for hypersonic flow simulations. The TVD algorithm uses a non-linear, minmod function as a flux limiter that maintains

Senior Research Engineer, Aerothermodynamics Branch, MS 408A, Fellow.

Senior Aeronautical Engineer, Aerothermodynamics Branch, MS 408A, Senior Member

1

American Institute of Aeronautics and Astronautics 
second-order accuracy away from extrema but can admit limit cycles in the convergence process, particularly in the vicinity of captured shocks. This occurrence usually manifests itself as a stalling of convergence at a very low error norm, essentially a benign ringing in the solution at a level that has no impact on aerothermodynamic quantities. Viscous flux is computed using central differences.

\section{Simulations}

All of the current simulations specify laminar flow of air in chemical nonequilibrium $\left(\mathrm{N}, \mathrm{O}, \mathrm{N}_{2}, \mathrm{O}_{2}, \mathrm{NO}\right)$ and thermal equilibrium. Only CFD point 1 is simulated for internal cavity flow simulations with freestream conditions: $\mathrm{V}_{\infty}=$ $7350.6 \mathrm{~m} / \mathrm{s}, \rho_{\infty}=3.900510^{-5} \mathrm{~kg} / \mathrm{m}^{3}, \mathrm{~T}_{\infty}=217.3 \mathrm{~K}, \alpha=40.1681$ deg., and time $=13.50 .53 .0$. The external flow simulations are implemented on the shared, baseline grid developed for the External Environments Team. The baseline grid has been modified to allow coupling of the external flow with flow through a breach in the wing entering a vented cavity. The following material is the first application of LAURA to flow in a vented cavity. Consequently, geometric complexity was incrementally added to better understand ensuing flow physics and effects of boundary condition specification.

Initially, quarter inch deep holes with varying diameters were inserted into the baseline, external flow solution at Panel 6 . (See Figure 1.) The boundary condition at the bottom of the hole used a zero-order extrapolation from interior points for density and velocity and a specified reservoir pressure equal to $2 \mathrm{p}_{\infty}$ (approximately $0.1 \mathrm{psf}$ ). The flow through this boundary evolved to be substantially supersonic for hole-sizes greater than two inches. The vent backpressure is sufficiently low to maintain at least sonic conditions through the vent and is characteristic of leeside levels.

The two-inch-hole simulation was then expanded to include an internal cavity extruded from the backside of panel 6 . The cavity was roughly $1 \mathrm{ft} \times 1 \mathrm{ft} \times 1 \mathrm{ft}$ but its shape evolved from an ad-hoc grid generation process. A two-inch vent hole was included at the far end of the cavity. Flow through the breach is fully coupled to the external flow and the vent boundary condition was transferred to its new location.

\section{A. Procedure}

An in-house utility to insert structured grids defining Reaction Control System (RCS) jets through the outer mold lines (OML) of hypersonic vehicles was modified to define

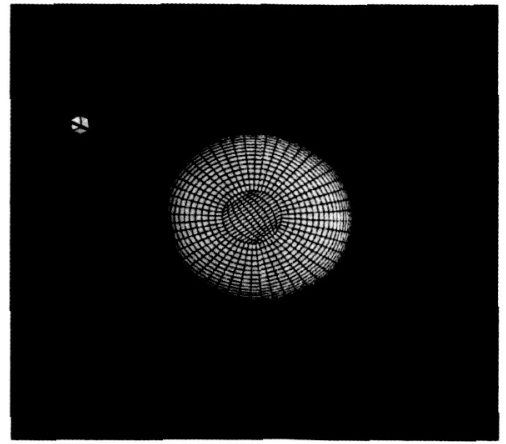

Figure 3: Structured grid within hole, approximately 20 cells deep and 60 cells across.

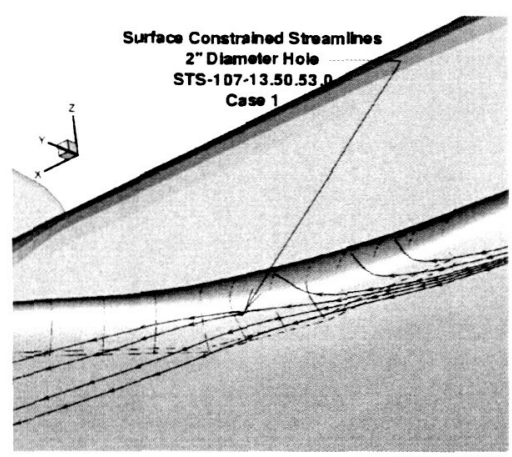

Figure 1: Streamlines over RCC panels on the leading edge and the location of the circular breach through the wing in panel 6.

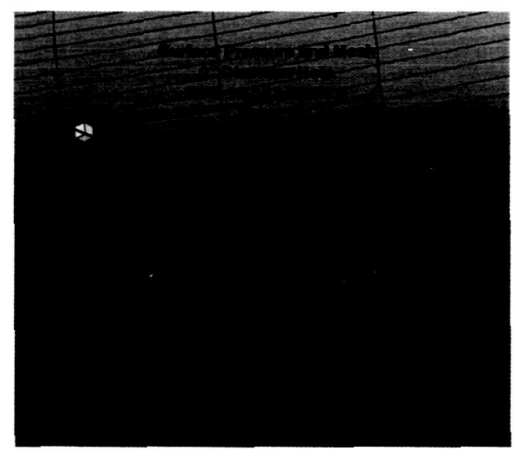

Figure 2: Modified surface grid in the vicinity of the hole.

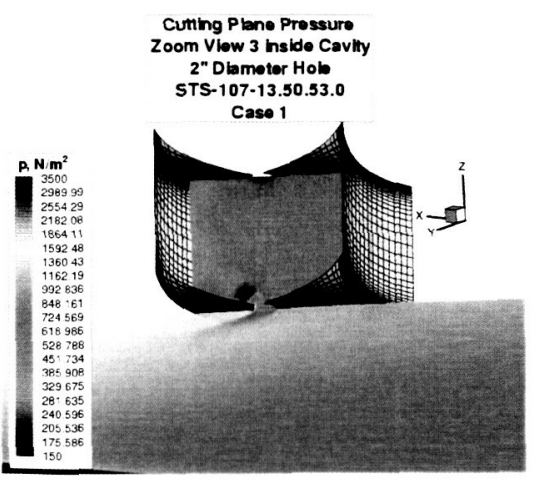

Figure 4: Pressure in cutting plane across breach and view of surface grid defining the 2 vented cavity.

American Institute of Aeronautics and Astronautics 
a quarter-inch deep, circular hole through the leading edge of shuttle orbiter wing. The modified surface grid blends smoothly with the baseline external grid as shown in Figure 2. A detail of the surface grid defining the hole is shown in Figure 3. This module has been developed further for Return to Flight applications to enable a more general grid and solution morphing capability around damage and repair sites.

Taking the inserted, rectangular domain surrounding the breach shown in Figure 2 and creating a quarter-inch offset orthogonal to the OML produced the near wall of the cavity. This surface was extruded into the interior in a direction defined by the axis of the hole. An initial cell size taken from the external flow domain was applied. A constant growth factor (1.20) grows the grid off the wall for 32 cells and then an inverse factor is applied for another 32 cells to resolve a boundary layer on the opposite wall. Sidewall boundary-layer resolution is achieved with a similar procedure in which additional points are added in directions defined by an extrapolated coordinate line. A view of this extruded cavity is shown in Figure 4.

\section{B. Immediate vent (No internal cavity)}

These simulations provided early evidence that the flow through a two-inch diameter (or larger) breach enters the cavity with significant retention of external flow directionality. A normal jet into the cavity was not an appropriate model for these conditions at CFD Point 1 (Mach 24). The breach diameters were of the same order or larger than the local, external boundary-layer thickness. High impingement heating and pressures on the downstream lip of the breach are computed. It is likely that hole shape would evolve as a slot cut in the direction of the external streamlines as shown in Figure 1. In the case of the six-inch diameter breach the boundary layer is fully ingested. Summary of results follow.

\section{B.1 Two-inch hole (Completed 03-28-03)}

The contour plot of total enthalpy in Figure 5 shows the boundary edge as the transition from orange to red color. The total enthalpy above the boundary layer edge, outside of viscous dissipation effects, is a constant. The boundary layer thickness is roughly $3 / 4$ of the hole diameter. The pressure field and streamlines entering the breach (Figure 6) indicate significant retention of streamwise momentum after processing by a Prandtl-Meyer expansion emanating from the sonic line in the boundary layer. Surface heating rates exceed $300 \mathrm{~W} / \mathrm{cm}^{2}$ (Figure 7) and surface temperatures approach $3000 \mathrm{~K}$ (Figure 8) at the downstream lip of

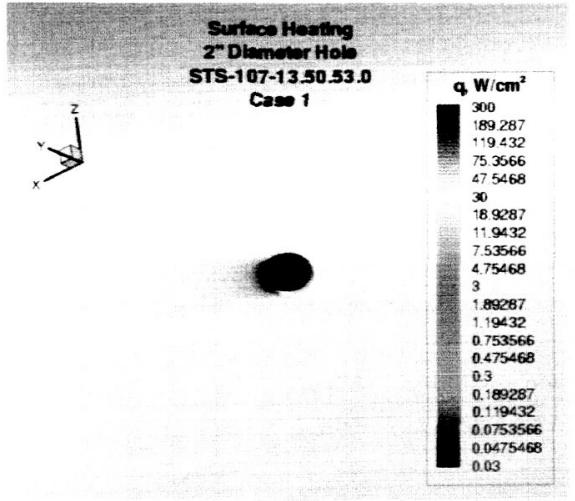

Figure 7: Surface-heating contours in the vicinity of the two-inch hole in Panel 6.

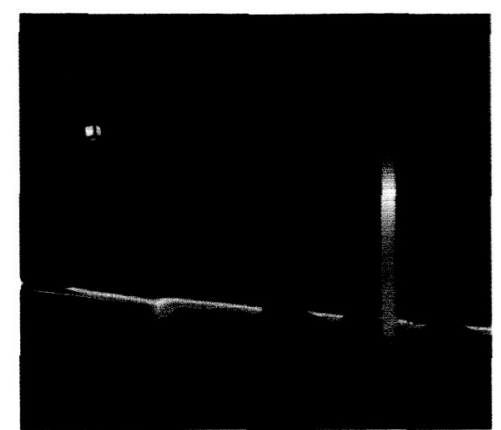

Figure 5: Total enthalpy in cut plane above hole showing partial ingestion of the boundary layer. (Blue jags are artifact of picture format conversion.)

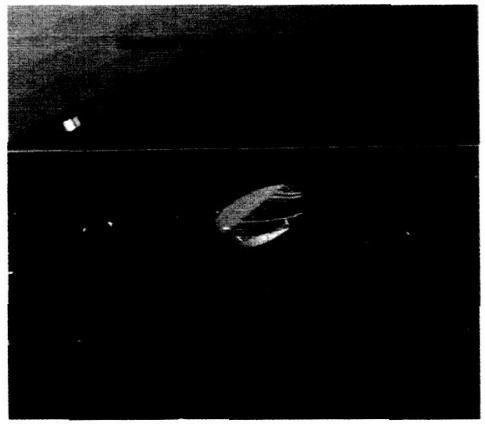

Figure 6: View of streamlines entering breach showing significant retention of external streamwise momentum.

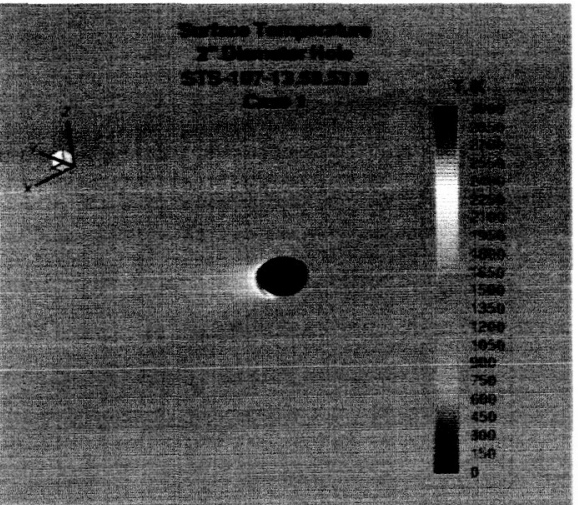

Figure 8: Surface temperature contours in vicinity of the two-inch hole in Panel 6.

3

American Institute of Aeronautics and Astronautics 
the breach where impingement is strongest. Ingested mass and energy rates are $0.000689 \mathrm{~kg} / \mathrm{s}$ and $9.92 \mathrm{~kW}$,

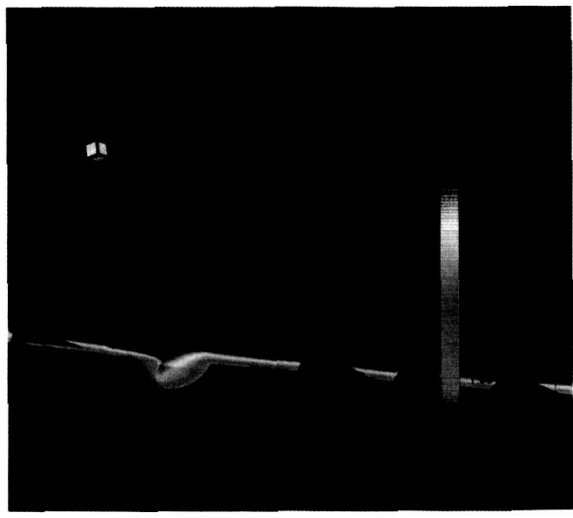

Figure 9: Total enthalpy in cut plane above fourinch hole showing significant ingestion of the boundary layer. (Blue jags are artifact of picture format conversion.)

respectively. Boundary conditions (radiative equilibrium, finite catalytic wall) and examination of near surface grid quality ( $\mathrm{Re}_{\text {cell }}$ of order 10 at impingement) suggest this heating rate is a lower bound. More data on grid convergence tests will be provided in the Panel 8 results to follow.

\section{B.2 Four-inch hole (Completed 03-31-03)}

The contour plot of total enthalpy in Figure 9 is repeated for the four-inch diameter hole. The boundary layer edge impinges on the downstream lip. The pressure field and streamlines entering the breach (Figure 10) indicate even more retention of streamwise momentum as compared to the previous case for the smaller hole. Surface heating rates again exceed $300 \mathrm{~W} / \mathrm{cm}^{2}$ (Figure 11) and surface temperatures exceed $3000 \mathrm{~K}$ (Figure 12) at the downstream lip of the breach where impingement is strongest. Ingested mass and energy rates are $0.00255 \mathrm{~kg} / \mathrm{s}$ and $46 \mathrm{~kW}$, respectively.

\section{B.3 Six-inch hole (Completed 04-01-03)}

The contour plot of total enthalpy in Figure 13 is repeated for the six-inch diameter hole. The boundary layer edge and some inviscid flow at the maximum freestream total enthalpy above it are completely ingested. The lip is impacted by streamlines carrying the total freestream enthalpy and a relatively large total pressure. The pressure field and streamlines entering the breach (Figure 14) show the same trends for retention of streamwise momentum as compared to the previous case for the four-inch hole. Surface heating rates again exceed $300 \mathrm{~W} / \mathrm{cm}^{2}$ (Figure 15) and surface temperatures exceed $3000 \mathrm{~K}$ (Figure 16) at the downstream lip of the breach where impingement is strongest. Of the three hole sizes, the present case should exhibit the largest heating because it is seeing undissipated

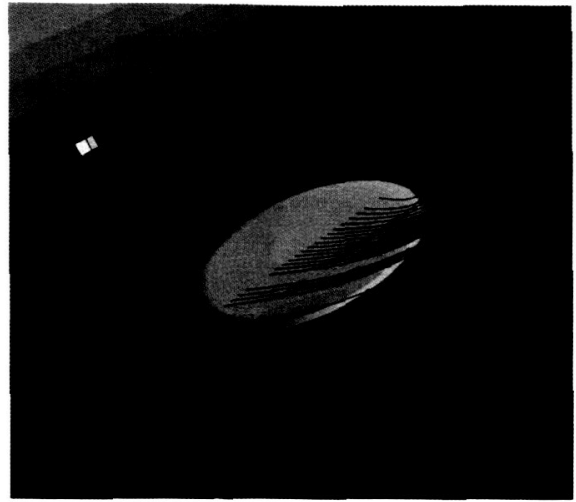

Figure 10: View of streamlines entering four-inch breach showing significant retention of external streamwise momentum.

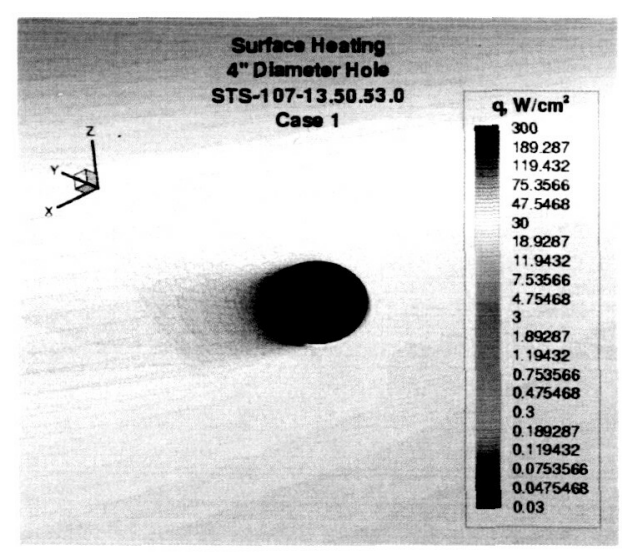

Figure 11: Surface-heating contours in the vicinity of the four-inch hole in Panel 6.

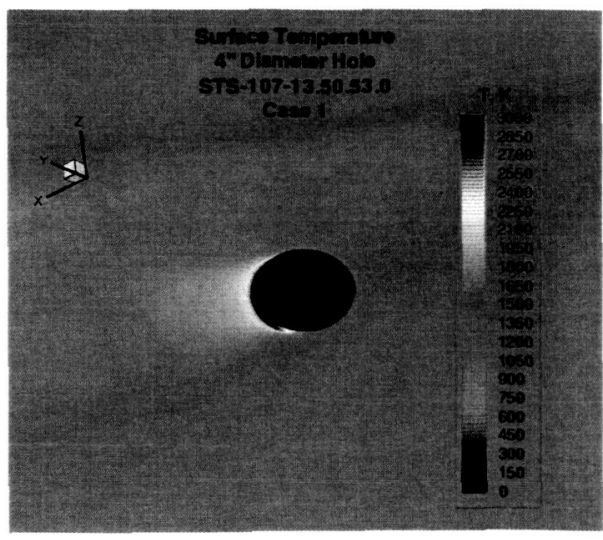

Figure 12: Surface temperature contours in vicinity of the four-inch hole in Panel 6. 


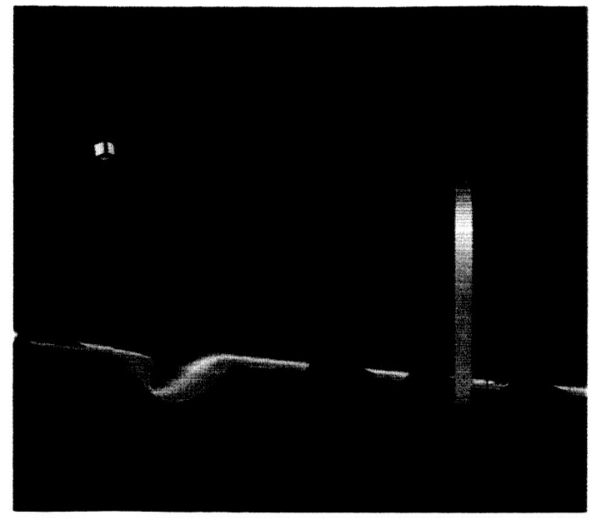

Figure 13: Total enthalpy in cut plane above six inch hole showing complete ingestion of the boundary layer. (Blue jags are artifact of picture format conversion.)

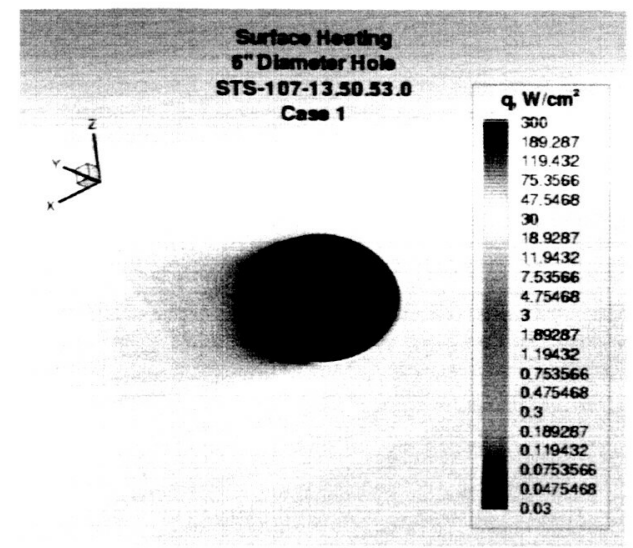

Figure 15: Surface-heating contours in the vicinity of the six-inch hole in Panel 6.

flow from above the boundary layer edge. Ingested mass and energy rates are $0.00566 \mathrm{~kg} / \mathrm{s}$ and $117 \mathrm{~kW}$.

respectively.

\section{Vented internal cavity}

These simulations allowed the jet to develop naturally within the cavity and to view initial impingement and deflections of flow to other surfaces. Jet orientation is a strong function of the hole depth to diameter ratio. Summary of results follow.

\section{C.1 Two-inch hole to vented box (Completed 04-10-03)}

Figure 17 shows streamlines entering the breach and the ensuing reflected wave pattern producing compressions and expansions in the jet core. The jet is non-orthogonal to the back plane of the breach. It has a significant directional component defined by the external flow direction. An expansion off the upstream side of the hole overtakes a shock off the downstream side of the hole.

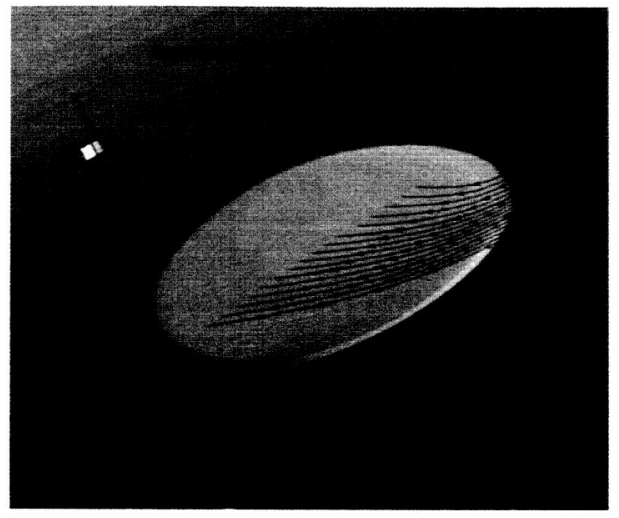

Figure 14: View of streamlines entering six-inch breach showing significant retention of external streamwise momentum.

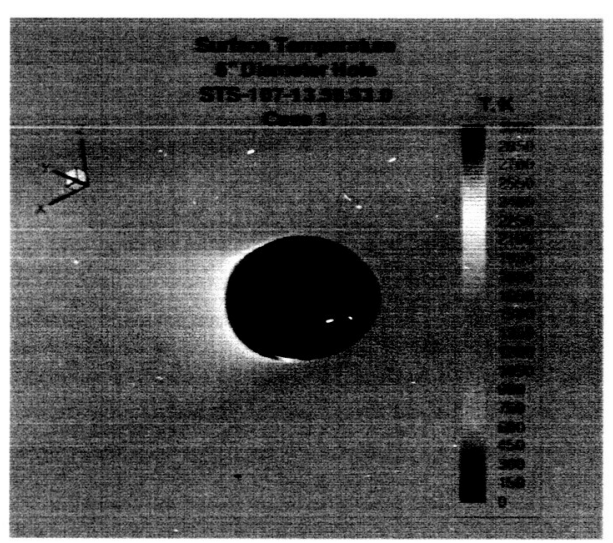

Figure 16: Surface temperature contours in vicinity of the six-inch hole in Panel 6.

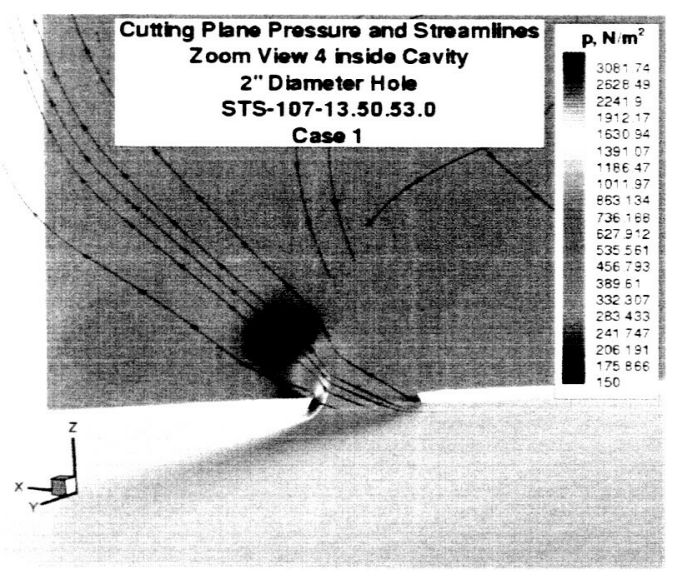

Figure 17: Streamlines entering the breach and pressure levels in the cutting plane. External flow direction is from the right to the left. 
The shock forms in response to supersonic flow from the upper portion of the boundary layer and the boundary layer edge impinging on the downstream lip. The Mach number distribution in Figure 18 provides additional detail of the jet entering the cavity. A sonic line sets up behind the inner edge of the breach lip shock indicating a choked condition. The interesting feature here is that the sonic line does not span the back plane of the hole but rather spans a smaller area defined by convergence of the streamlines entering from the circular border. Pressure coefficients in Figure 19 indicate the ambient pressure level in the cavity of approximately (1/8) $\rho_{\infty} \mathrm{V}_{\infty}{ }^{2}$. This level is attained at the back plane of the hole. The dependence of cavity ambient pressure on vent hole size has not been explored. It is interesting to note that a specification of the vent pressure immediately behind the back plane of the hole results in approximately the same ambient pressure level in the

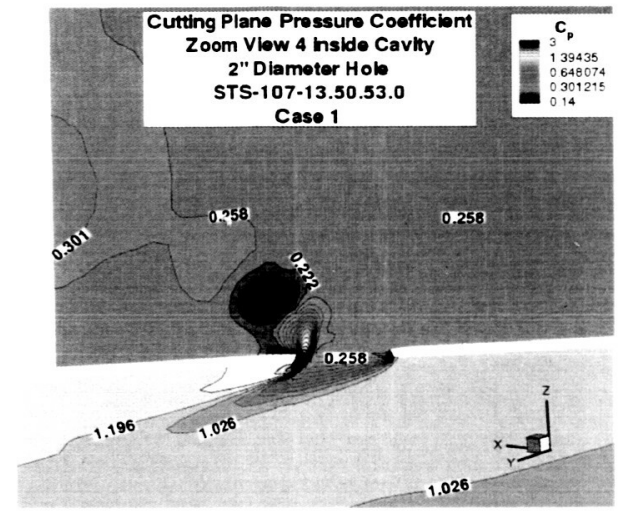

Figure 19: Pressure coefficient in vicinity of the breach.

cell preceding the outflow boundary. Pressures exceeding the external pressure of approximately $(1 / 2) \rho_{\infty} V_{\infty}{ }^{2} \quad\left(C_{p}=1\right)$ would effectively block ingestion of external flow.

The temperature in the shock over the downstream lip approaches $8000 \mathrm{~K}$ in Figure 20. The corresponding total enthalpy profile is shown in Figure 21 that corresponds to the earlier result in Figure 5 except now the cavity beneath the hole is included. Conditions just below the boundary layer edge impact the lip to drive the large surface heating rates and surface temperatures (Figure 22). The impingement heating on the edge will tend to cut a slot along the surface in the external streamline direction.

The oxygen content of ingested flow is almost fully dissociated as indicated in Figure 23. A strong catalytic heating effect would be expected on metallic cavity walls but the finite catalytic boundary condition on the external surfaces was continued into the cavity. Also, the radiative equilibrium wall boundary condition assumes cavity walls can radiate to free space whereas the actual closed environment would restrict radiative cooling. Conductive cooling through metallic walls is not considered. Given these conditions (expected to produce lower heating rates than actually encountered in flight) the surface temperatures on the cavity walls (Figure 24) already exceed the melting point of pure aluminum at the impingement zone. Note that the breach hole through the lower surface is not visible in this figure. The vent hole on the top surface still sees temperatures that would melt aluminum.

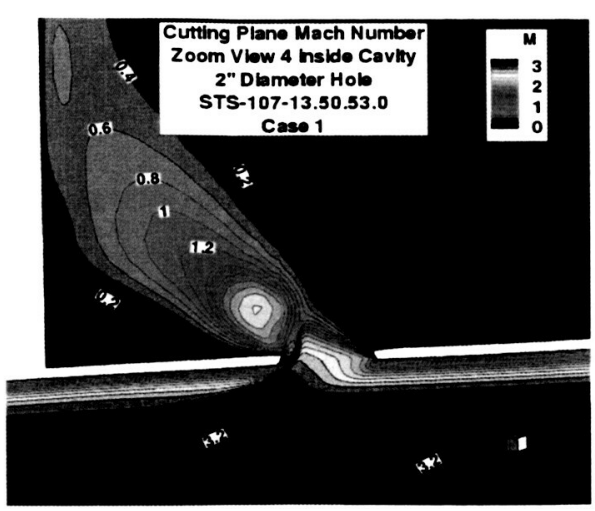

Figure 18: Mach number contours across the breach and in the jet entering the cavity.

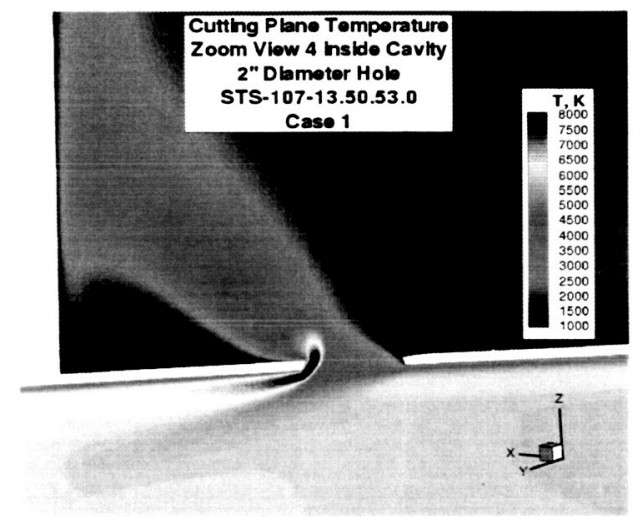

Figure 20: Temperature in vicinity of the breach.

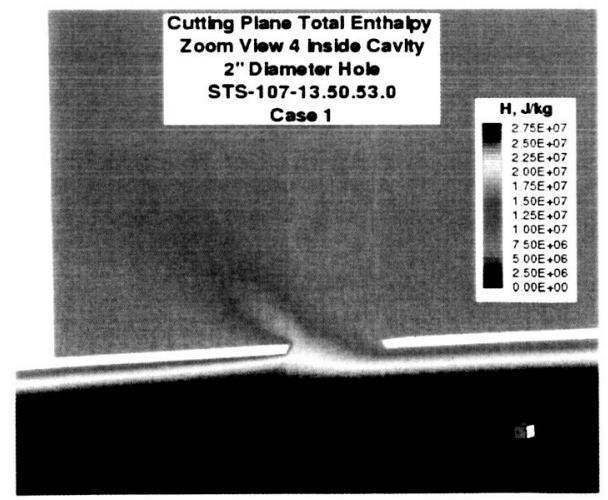

Figure 21: Total enthalpy in vicinity of breach indicating level of ingestion of the external boundary layer. 


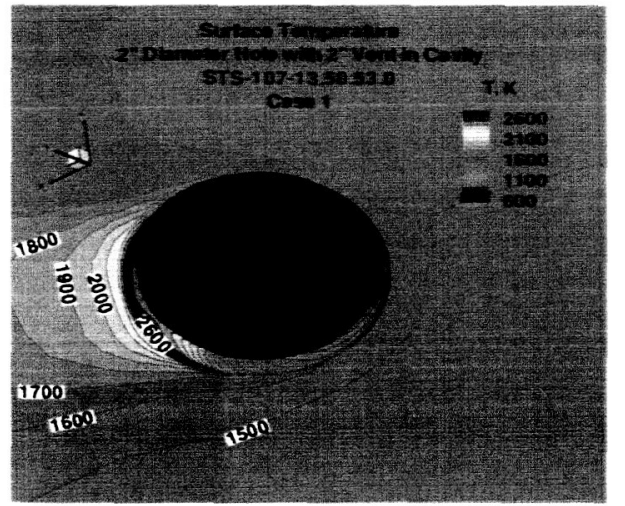

Figure 22: Surface temperatures in vicinity of downstream lip of two-inch hole over cavity.

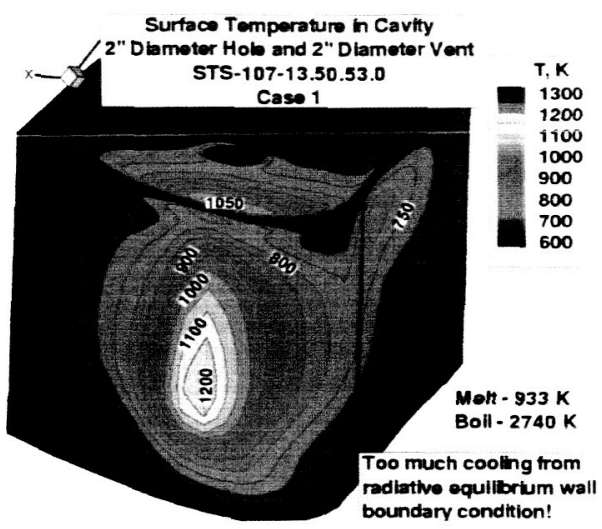

Figure 24: Surface temperature on cavity walls with identical cooling mechanisms as used for external flow.

The vented cavity mass and energy flux through the bottom of the hole are $6.610^{-4} \mathrm{~kg} / \mathrm{s}$ and $8.8 \mathrm{~kW}$, respectively. These values are $4 \%$ and $12 \%$ smaller than the corresponding rates for a two-inch hole venting directly to a constant pressure reservoir reported previously. Peak heating on the downstream lip of the hole into the cavity is $255 \mathrm{~W} / \mathrm{cm}^{2}$ compared to $313 \mathrm{~W} / \mathrm{cm}^{2}$ when the constant-pressure reservoir boundary condition is used. The average pressure in the cavity is larger than the venting pressure level and tends to reduce mass and energy ingestion rates as compared to the more conservative direct vent boundary condition.

\section{C.2 1/4-inch hole to vented box (Completed 04-28-03)}

A quarter-inch diameter hole was simulated to provide reference conditions in which the hole diameter was not larger than the hole depth and all hole dimensions were smaller than the local boundary layer thickness. In this case, the jet develops in an orthogonal direction to the wall as seen in the Mach number contour of Figure 25 Impingement heating and temperatures on the downstream lip are much more benign (Figure 26) because the energy

7

American Institute of Aeronautics and Astronautics
Figure 23: Atomic oxygen mass fraction ingested through breach.

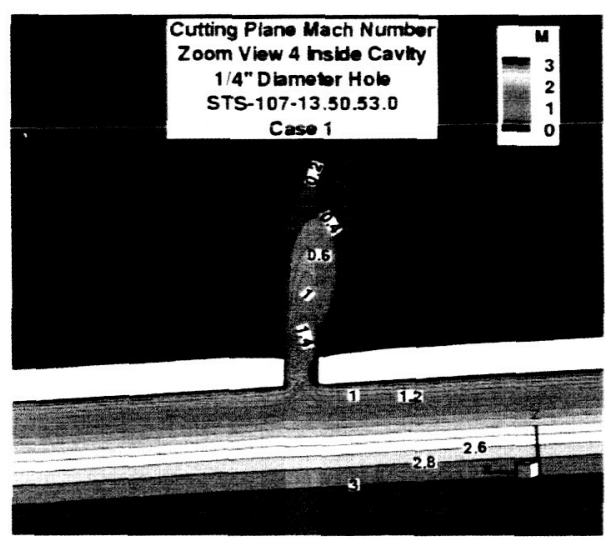

Figure 25: Mach number contours of jet entering cavity through quarter-inch diameter hole.

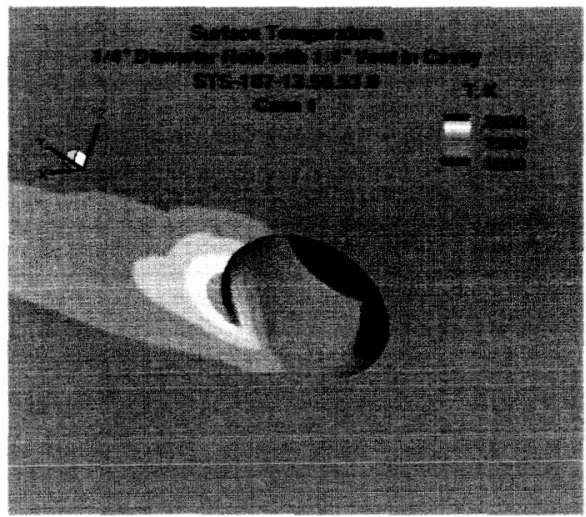

Figure 26: Surface temperatures in vicinity of ouarter-inch hole. 
content and total pressure of the impinging streamlines emerging from deep within the boundary layer is significantly dissipated. Temperatures entering the cavity (Figure 27) exceed $1000 \mathrm{~K}$.

\section{Ten-inch Hole in Panel 8}

These simulations were initiated in mid-May to complement ongoing simulation work on higher fidelity internal grids and to insure that sufficient corroborating data for engineering analyses could be made available as quickly as possible. However, greater fidelity to the internal geometry was requested (as compared to the adhoc cavity) so that jet development lengths and splash to adjoining internal surfaces may be better simulated.

A simulation plan was developed to maximize productivity in the limited, remaining time. First, the simulation of a ten-inch hole in Panel 8 into the ad-hoc cavity as described in the previous section was produced. (The grid in this case had to be post-processed by the VGM utility to compensate for problems associated with the larger ratio of local effective radius to hole diameter.) This simple simulation would next be used to initialize the cavity flow in a more realistic "chunnel" geometry generated concurrently. A grid sequencing process is used to complete the simulation and significant sharpening of the jet was observed in the transition from moderate to fine grid. When the jet boundaries sharpened, it was noted that the impingement location was underresolved. An additional grid-processing step was required to bring a factor ten finer resolution on the wall at impingement.

The chunnel geometry was built off of the ad-hoc cavity and expanded to include the domain behind panels 7-9. The back plane was defined by the actual spar location. Two vent slots with combined area of $66 \mathrm{in}^{2}$ were included on the leeside, back surface. An internal core system of blocks was approximately bounded by the inner extent of ribs at the panel interfaces to accommodate possible future extensions of internal complexity. A combination of an O- and C-grid topology was used to accommodate the complex internal structure. The C-grid provides the ability to capture the rib and spar geometry while offering a decoupling of the grid requirements for stretching and clustering at the internal wall surfaces. As shown in Figure 28 and Figure 29, the C-grid easily captures the necessary chunnel features. Within the C-grid, in the bottom block, the hole in the RCC is accommodated by simply replacing the block of the $\mathrm{C}$-grid with an "embedded" O-grid, which enables accurate modeling of the flow entering the chunnel. The $\mathrm{O}$ - and $\mathrm{C}$-grid junction enables the accurate modeling of the existing geometry while offering the expandability to increasing geometric complexity without restructuring and regenerating the entire chunnel volume grid.

In the final simulation, the top vents were closed and the side core vents (combined area $79.3 \mathrm{in}^{2}$ ) were opened to enable the splash from an impinging jet to spill over a rib and through a vent. Summary of results follow.

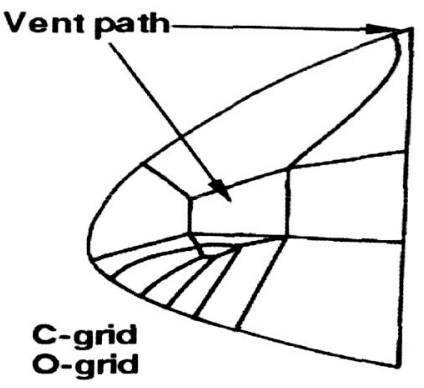

Figure 28: Internal block structure merging ad hoc cavity and chunnel showing relative vent positions.

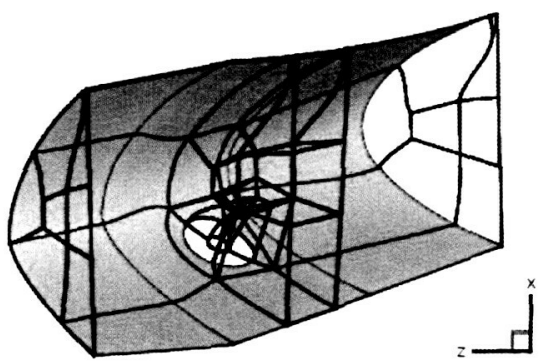

Figure 29: Global view of chunnel block structure

American Institute of Aeronautics and Astronautics 


\section{D.1 Vented internal box (Completed 05-19-03)}

Mach number and streamlines entering the box through the ten-inch hole in panel 8 are shown in Figure 30. Streamlines exit a ten-inch diameter vent at the top of the box. The flow is strongly supersonic entering this cavity. The most interesting details of this simulation (that remain true for the subsequent chunnel simulations) are the high temperatures and pressures in the shock layer over the downstream lip of the hole. The high temperature (approaching $12000 \mathrm{~K}$ in Figure 31) is easily explained because the lip is bathed in flow that carries the full freestream total enthalpy. The local pressure coefficient $\left(C_{p}\right.$ $=10$ ) is higher than the stagnation point because the local streamlines here were processed by an oblique shock so that total pressure losses are less than those experienced in crossing a normal shock. Computed heating rates exceed $800 \mathrm{~W} / \mathrm{cm}^{2}$ in this case. When considering the factor of increase in pressure and factor decrease of local radius of curvature relative to the stagnation point one may expect this rate (ignoring ablative cooling) is a lower bound.

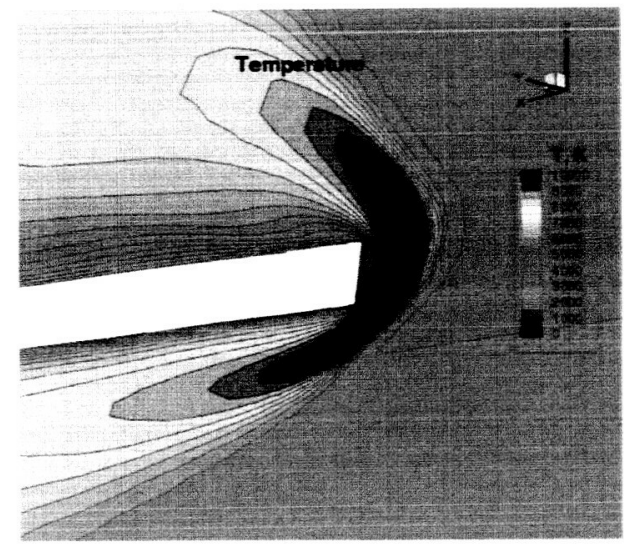

Figure 31: Details of temperature in shock layer over downstream lip with ten-inch breach through panel 8 .

\section{D.2 Chunnel with slotted leeside vents (Completed 06-01-} 03)

Results in this section discuss the solution after adaptation of the finest grid to concentrate more mesh to the jet impingement point. Error norms had dropped to order 0.4 (a five to six order of magnitude decrease from the initial solution off the coarse grid). Some unsteadiness is evident when monitoring the solution but there was insufficient time to gather meaningful statistics on frequency. The jet impingement point has moved very little over about 10000 relaxation steps and it is believed that the basic flow physics presented here are correct.

Details of the jet in the cutting plane through the chunnel are presented in Figure 32 (total enthalpy), Figure 33 (Mach number), and Figure 34 (temperature). A tongue of high enthalpy flow persists up to the impingement point. A

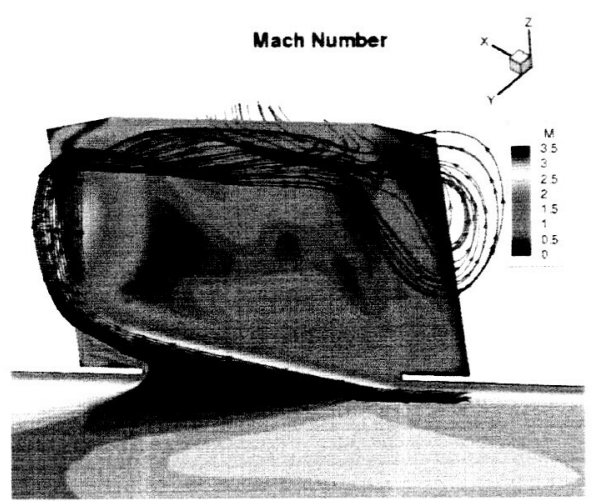

Figure 30: Mach number and streamlines in cavity behind panel 8 with ten-inch diameter hole and ten-inch diameter vent.

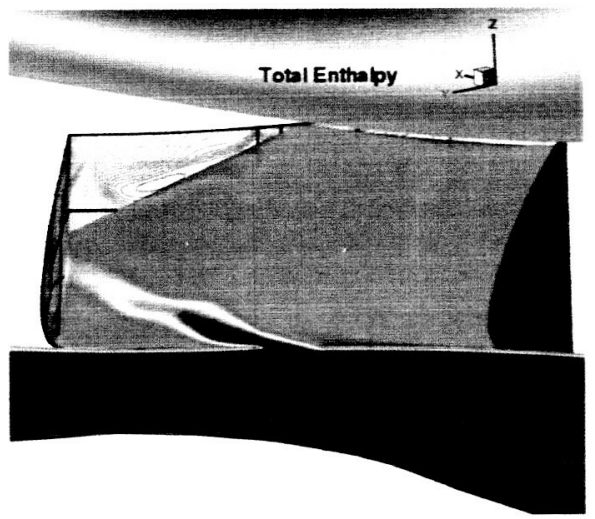

Figure 32: Total enthalpy entering chunnel through Panel 8. Venting is through slots on top of chunnel.

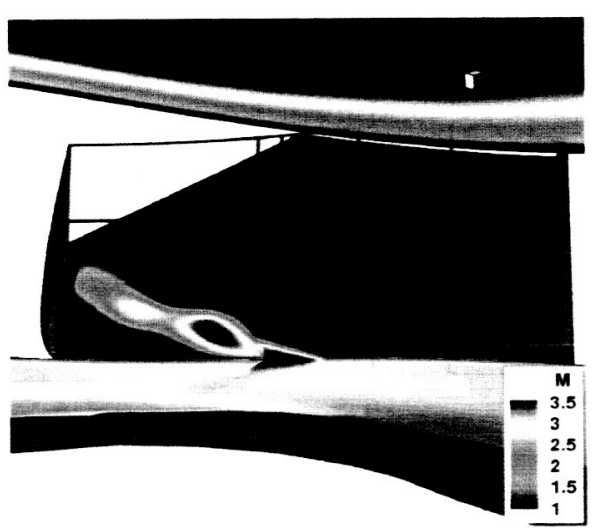

Figure 33: Mach number contours focused on supersonic domain. 
high temperature shock layer forms above this location. The jet initially scrapes the surface and then rises above it.

A "strip" of high pressure (Figure 35) and high heating rate (Figure 36 ) is evident at the relatively narrow impingement location. These figures show the sharpening of the profile associated with refinement from the medium grid to the fine grid (a factor of 2 in the $\mathrm{i}, \mathrm{j}$, and $\mathrm{k}$ directions). Review of solution metrics at the impingement showed high cell Reynolds number and large jump between imposed wall temperature $(500 \mathrm{~K})$ and boundary cell center temperature $(2880 \mathrm{~K})$. Consequently, a grid adaptation was implemented to provide a target factor of 10 finer grid at the wall (idirection) using a greater stretching factor for the same number of cells in the near wall blocks. Even with this refinement, the worst-case cell center temperature next to the wall was $(1160 \mathrm{~K})$. The convergence of peak impingement heating on these three sequential cases (not located at exactly the same surface mesh point) progressed from $116 \mathrm{~W} / \mathrm{cm}^{2}$ (medium grid) to $301 \mathrm{~W} / \mathrm{cm}^{2}$ (fine grid) to $721 \mathrm{~W} / \mathrm{cm}^{2}$ (adapted fine grid, Figure 37 )

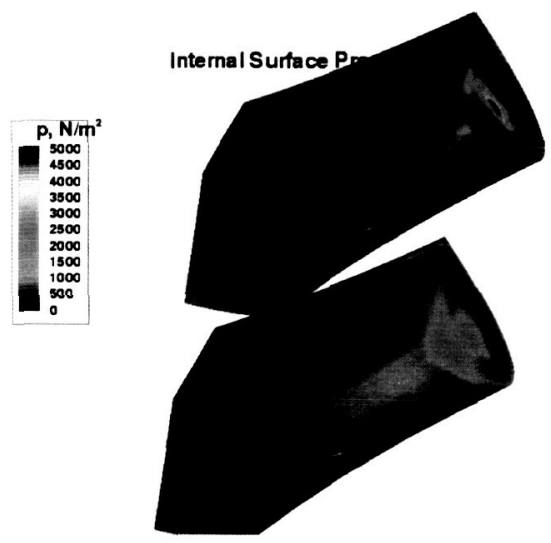

Figure 35: Comparison of chunnel surface pressures from medium grid and unadapted fine grid.

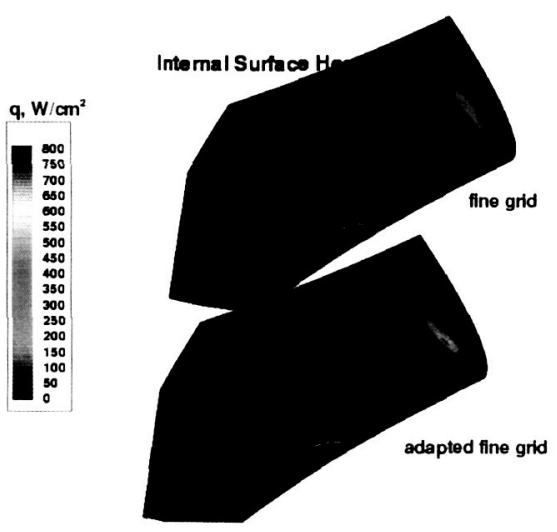

Figure 37: Comparison of chunnel surface heating from unadapted fine grid and adapted fine grid on impingement surface.

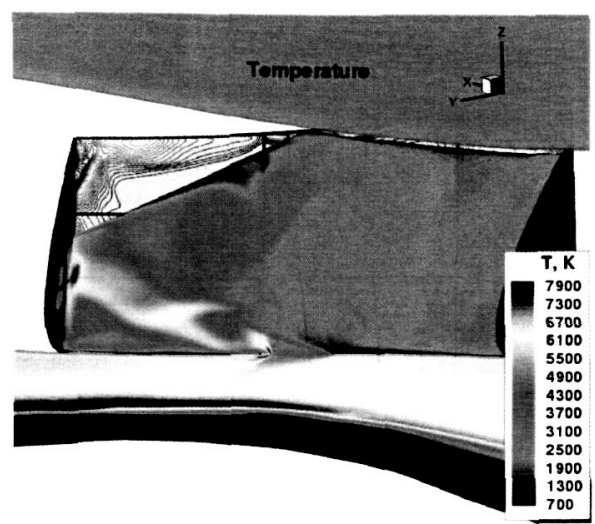

Figure 34: Temperatures in cutting plane through chunnel showing high temperatures at impingement of jet on the side wall.

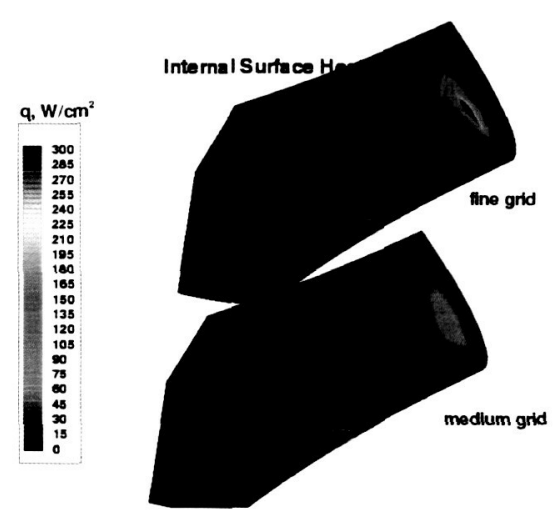

Figure 36: Comparison of chunnel surface heating from medium grid and unadapted fine grid.

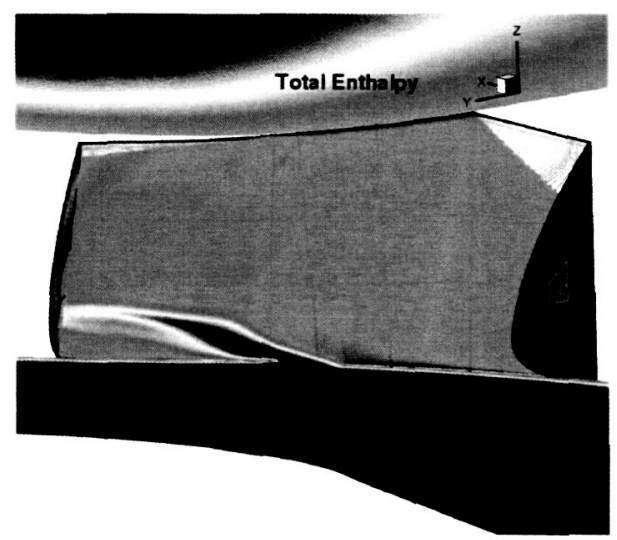

Figure 38: Total enthalpy in chunnel with side vents. 


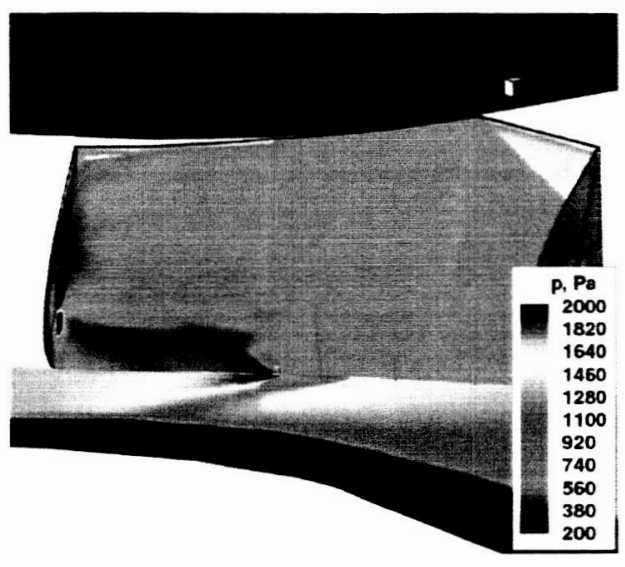

Figure 39: Pressure in chunnel with side vents.

\section{D.3 Chunnel with side vents (Completed 06-06-03)}

This side vent case (top vents now closed) was initialized from the previous top vent case with the finest grid and adaptation of grid on the impingement boundary.

The error norm for this case jumped about 4 orders of magnitude from the previous converged solution and returned to order 1 values at this "snapshot". An attempt to force the solution to advance more quickly with a constant, large time step eventually diverged. A smaller constant time step was again applied and the solution began to recover but insufficient time remained to drive it to convergence. The solution posted here is thought to have a jet impingement point that will continue to rise toward the side vent. The magnitude of jet oscillations cannot be estimated from the solution generated to date. A representative jet structure is presented in Figure 38 (total enthalpy) and Figure 39 (pressure). The basic structure is very similar to that observed in the previous case. The expansion process to sonic conditions at the side vent is displayed in Figure 40 (pressure) and Figure 41 (Mach number) in the impingement zone.

Top vent versus side vent internal surface pressures are compared in Figure 42. The equivalent heating comparison is presented in Figure 43 and Figure 44. Heating at impingement on the finest grid has remained between 500 and $700 \mathrm{~W} / \mathrm{cm}^{2}$.

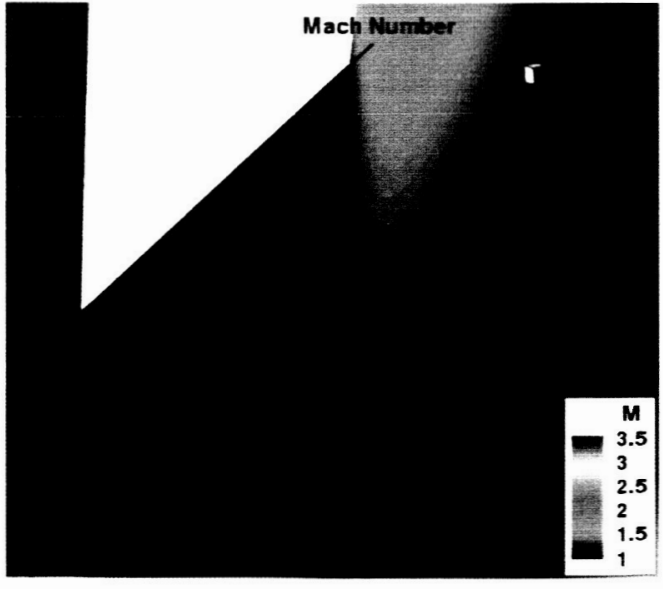

Figure 41: Magnified view of Mach number expanding to sonic condition at side vent.

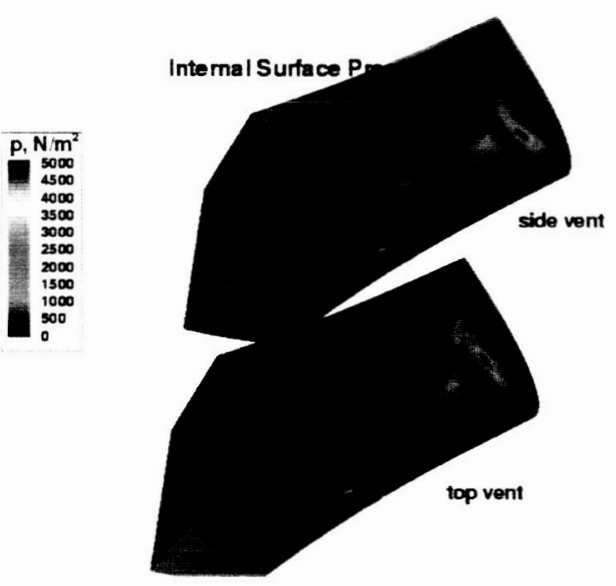

Figure 42: Comparison of chunnel surface pressures with top and side vents. 


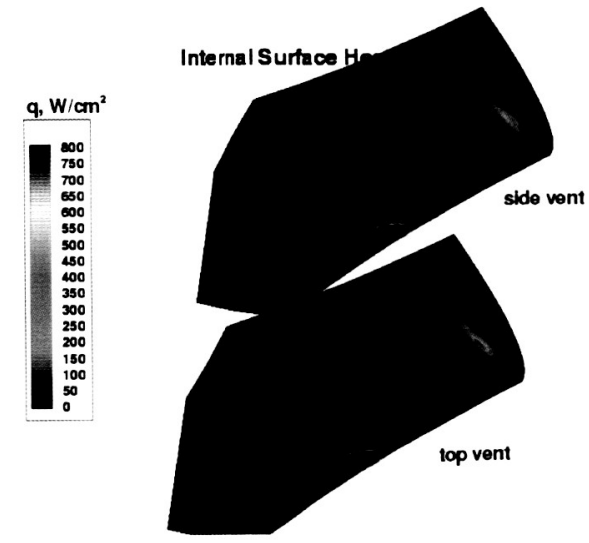

Figure 43: Comparison of chunnel surface heating with top and side vents.

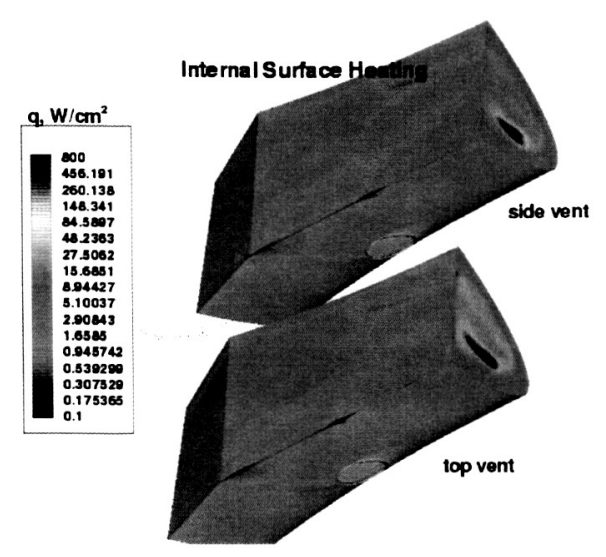

Figure 44: Logarithmic scaling of surface heating.

\section{Summary}

A baseline solution for CFD Point 1 (Mach 24) in the STS-107 accident investigation was modified to include effects of holes through the leading edge into a vented cavity. The simulations were generated relatively quickly and early in the investigation because simplifications were made to the leading edge cavity geometry and an existing utility to merge scarfed nozzle grid domains with structured baseline external domains was implemented. These simplifications in the breach simulations enabled: (1) a very quick grid generation procedure; and (2) high fidelity corroboration of jet physics with internal surface impingements ensuing from a breach through the leading edge, fully coupled to the external shock layer flow at flight conditions.

These simulations provided early evidence that the flow through a two-inch diameter (or larger) breach enters the cavity with significant retention of external flow directionality. A normal jet directed into the cavity was not an appropriate model for these conditions at CFD Point 1 (Mach 24). The breach diameters were of the same order or larger than the local, external boundary-layer thickness. High impingement heating and pressures on the downstream lip of the breach were computed. It is likely that hole shape would evolve as a slot cut in the direction of the external streamlines. In the case of the six-inch diameter breach the boundary layer is fully ingested. The boundary-layer edge impinges on the downstream lip of a four-inch diameter hole. The boundary layer is not fully ingested for a two-inch diameter hole but significant retention of the external flow momentum is evident in the jet. Only the bottom part of the boundary layer is ingested through a quarter-inch diameter hole and the ensuing jet into the cavity is nearly orthogonal to the mold line.

\section{References}

\footnotetext{
${ }^{1}$ Gnoffo, P. A.; Gupta, R. N.; and Shinn, J.: "Conservation Equations and Physical Models for Hypersonic Air Flows in Thermal and Chemical Nonequilibrium," NASA TP 2867, 1989.

${ }^{2}$ Cheatwood, F. M. and Gnoffo, P. A. "Users Manual for the Langley Aerothermodynamic Upwind Relaxation Algorithm (LAURA)," NASA TM 4674, April 1996.

${ }^{3}$ Weilmuenster, K. J.; Gnoffo, P. A.; Greene, F. A.: "Navier-Stokes Simulations of Orbiter Aerodynamic Characteristics Including Pitch Trim and Bodyflap," Journal of Spacecraft and Rockets, Vol. 31, No. 3, May-June 1994, pp355-366.

${ }^{4}$ Gnoffo, P. A.; Weilmuenster, K. J.; Alter, S. J.: "Multiblock Analysis for Shuttle Orbiter Re-entry Heating from Mach 24 to Mach 12," Journal of Spacecraft and Rockets, Vol. 31, No. 3, May-June 1994, pp367-377.

${ }^{5}$ Kleb, W. L.; Weilmuenster, K. J.: "Characteristics of the Shuttle Orbiter Leeside Flow During a Re-Entry Condition," Journal of Spacecraft and Rockets, Vol. 31, No. 1, May-June 1994, pp8-16.

${ }^{6}$ Orbiter Experiments (OEX) Aerothermodynamics Symposium, Edited by David Throckmorton, NASA

Conference Publication 3248, Part I and II, April 1995.

${ }^{7}$ Roe, P. L.: “Approximate Riemann Solvers, Parameter Vectors, and Difference Schemes," Journal of Computational Physics, Vol.43, Oct. 1981, pp357-372.

${ }^{8}$ Yee, H. C.: "On Symmetric and Upwind TVD Schemes," NASA TM-88325, June 1986.
} 